\title{
PROPERTIES OF ION TEMPERATURE GRADIENT DRIFT INSTABILITIES IN H-MODE PLASMAS
}

\author{
T.S. HAHM AND W.M. TANG \\ Princeton Plasma Physics Laboratory \\ P.O. Box 451 \\ Princeton, New Jersey 08543
}

\begin{abstract}
Experimental results from tokamaks such as DIII-D and JET have indicated that the electron density profile in H-mode ("high-confinement") discharges can be nearly flat over most of the plasma, and, in some cases, even inverted (outwardly peaked). These conditions have very interesting implications fur pictures of anomalous thermal transport based on the presence of jon temperature gradient $\left(\eta_{i}\right)$ drift instabilities. The present paper includes (i) a new derjvation of the ion temperature gradient threshold for weak density gradient $\eta_{i}$ modes when ion transit resonances are taken into account; and (ii) the first derivation of threshold conditions for the onset of $\eta_{i}$-modes when $\eta_{i}$ is negative along with the properties of these negative $\eta_{i}$ instabilities when the thresholds are exceeded. Possible consequences for confinement in $\mathrm{H}$-mode plasmas are discussed.
\end{abstract}




\section{INTRODUCTION}

One of the most important problems currently encountered in tohamak research involves understanding the physics responsible for the favorable confinement properties observed in so-called $\mathrm{H}$-mode discnarges. For example, design studies for future ignition experiments such as CIT (Compact Ignition Torus) have is licated that acceptable performance levels in such auxiliaryheated divertor plasmas will require maintaining $\mathrm{H}$-mode rather than the more common L-mode-type ("low" mode) energy confinement trends. A!though the energy confinement time in $\mathrm{H}$-mode plasmas is typically observed to be about a factor of 2 better than L-mode cases, the thermal transport nevertheless remains very anomalously large. Hence, confinement pictures based on the presence of drift-type microinstabilities such as ion temperature gradient $\left(\eta_{i}\right)$ modes continue to be plausible.

Previous theoretical and experimental studies have supported the proposition that $\eta_{\mathrm{i}}$-type drift instabilities ${ }^{1-3}$ can account in large part for the observed non-neoclassical features of ion thermal losses in high density ohmic discharges as well as auxiliary-heated L-mode plasmas. ${ }^{4-9}$ The improved confinement in pellet-fueled high density ohmic plasmas in the ALCATOR C. TFTR, ASDEX, and TEXT tokamaks has been shown to be consistent with results from transport codes which include models for both trapped-electron and $\eta_{i}{ }^{-t y p e} d r i f t$ instabilities. ${ }^{t-7,9}$ More direct suppurting physical evidence has come from niuctuation measurements carried out on the TEXT tokamak. Results have indicated (i) the appearance of an ion feature in the spectrum of the bigh density, gas-fueled cases where the $\eta_{i}$-nodes are predicted to be present and (ii) the absence of such a feature in the improved confinement pellet-fueled cases where the $\eta_{i}\left(\equiv d \ln T_{1} / d \ln n_{i}\right)$ parameter is inferred to fall below the stability threshold (i.e., $\eta_{i}<\eta_{i c}$ ). ${ }^{9}$ Although uncertainties in the profile measurements preclude definitive comparisons with specific threshold conditions, the qualitative trends noted in these studies ${ }^{8,9}$ are indeed very suggestive that the $\eta_{i}$-modes could be present.

With regard to $\mathrm{H}$-mode plasmas, experimental results from tokamaks such as DIII-D $D^{10}$ and JET ${ }^{11}$ have characteristic electron density profiles which can be nearly tlat over most of the discharge and can even be outwardly peaked in some instances. For the weak density gradient (very large $\eta_{i}$ ) limit, it has been previously pointed out that a relatively weak form of toroidal $\eta_{2}$ insta- 
bilities could be responsible for the improved but still strongly anomalous thermal losses observed. ${ }^{12}$ Onset of instability in these cases is independent of the density gradient scale length $\left(L_{n}\right)$ and requires only a sufficiently steep ion temperature gradient. ${ }^{12-16}$ In the present paper attention is focused on situations where $\eta_{k}$ is negative. This scenario $\left(\eta_{i}<0\right)$ is particularly relevant to [f-mode plasmas because electron density profiles on DIII-D. for example. have been observed to be relatively flat, while the corresponding $Z_{\text {es }}$ profiles appear to be centrally peaked. ${ }^{17}$ Moreover, H-mode discharges with outwardly peaked electron density profiles have been measured in both the DIII-D ${ }^{10}$ and the JET ${ }^{11}$ tohamaks. Wotivated by these considerations, we have proceeded to derive for the first time the onset conditions for negative $\eta_{1}$ modes. The properties of these instabilities are quite diferent from those of ion temperature gradient modes either with $\eta_{i}>0$ or with flat ion density gradients $\left(\eta_{i} \rightarrow \infty\right)$. Specifically, since $\left|\gamma / \omega_{r}\right| \ll 1$ (with $\gamma$ and $\omega_{r}$ being the growth rate and real frequency) for these instabilities. even away from the threshold, it can now be argued that a weak turbulence analysis of the resultant confinement characteristics is reasonable.

The remainder of this paper is organized as follows: In Sec. II the threshold conditions and basic characteristics of $\eta_{i}$-modes are derived in a sheared slab geometry with ion transit resonances included for normal $\left(\eta_{i}>0\right)$, flat $\left(\eta_{i} \rightarrow \infty\right)$. and inverted $\left(\eta_{i}<0\right)$ density profiles. The features of the negative $\eta_{i}$ modes are compared with those of the familiar positive $\eta_{i}$ instabilities, and both analytic and numerical results are presented here. The possible role of these instabilities in H-mode plasmas is discussed in Sec. III, and a brief summary of the main conclusions of the paper are given in Sec. IV.

\section{ANALYSIS OF LINEAR PROPERTIES OF $\eta_{i}$-IN- STABILITIES}

The instability threshold conditions for ion temperature gradient drift modes are derived in this section. We consider a sheared slab geometry and construct a theoretical model consisting of the Boltzmann response for electrons and a collisionless gyrokinetic ion response which includes waveparticle transit resonances. This corresponds to the frequency ordering, $k_{\| \mid} v_{t e} \gg \omega \sim k_{||} v_{t,}$ with $k_{\|}$being the parallel wave number and $v_{t,}$ being the thermal velocity of species $j$. Long to moderate perpendicular wavelength 
modes $\left(k_{\perp}^{2} \rho_{i}^{2}<1\right)$, which would most strongly infuence thermal transport. are considered. In particular, the long wavelength modes $\left[k_{y}^{2} \rho_{i}^{2} \lesssim\left(L_{T_{1}} / R\right)^{1 / 2}\right]$ are predominantly sound-wave-like in nature and are well extended along the magnetic field line. Basically, the mode structure is "flute-like" without significant 'ballooning' to the outside unfavorable curvature region. Hence. toroidal curvature modifications to the sheared slab results here are relatively minor. ${ }^{13.18}$

The well-known eigenmode equation ${ }^{19}$ is obtained by imposing the quasineutrality condition on the electron ion density responses; i.e..

$$
\frac{d^{2}}{d x^{2}} \phi+Q(x) \phi=0
$$

with

$$
Q(x)=-b_{3}+\tau \frac{\tau+1+\left(\tau+\frac{1}{\Omega}-\frac{\eta_{1}}{2 \Omega}\right) Z_{1} \xi_{1}+\frac{\eta_{1}}{\Omega} \xi_{1}^{2}\left(1+Z_{1} \xi_{1}\right)}{\left(\tau+\frac{1}{\Omega}+\frac{\eta_{1}}{2 \Omega}\right) Z_{3} \xi_{i}+\frac{\eta_{1}}{\Omega} \xi_{i}^{2}\left(1+Z_{i} \xi_{2}\right)}
$$

where $x=\left(r-r_{0}\right) / \rho_{s}, \Omega=\omega / \omega_{m e}, \xi_{z}=\omega / k_{\|} v_{t i}, r=T_{e} / T_{n}, b_{s}=k_{y}^{2} \rho_{s}^{2}$, and $Z_{1}$ is the usual plasma dispersion function for ions with argument $\xi_{i}$. The second order differential equaiion, Eq. (1), is valid when $\rho_{i}^{2}\left(k_{x}^{2}+k_{y}^{2}\right)<1$. In analyzing Eq. (1), the usual procedure is to first consider the fluid ion limit where $|\omega| \gg\left|k_{\|}\right| v_{\mathrm{e} i}$ is satisfied. Using the large-argument $\left\langle\xi_{1}\right)$ asymptotic form of the Z-function in Eq. (1), we obtain,

$$
\frac{\partial^{2}}{\partial x^{2}} \phi+\left[-b_{s}+\frac{1-\Omega}{\Omega+\frac{i+\Omega_{1}}{\tau}}+\left(\frac{L_{n}}{L_{s}}\right)^{2} \frac{x^{2}}{\Omega^{2}}\right] \phi=0 .
$$

This Weber equation then yields the following dispersion relation:

$$
\left(1+b_{s} i \Omega^{2}+\left(b_{s} \frac{1+\eta_{i}}{\tau}-1+i \frac{L_{n}}{L_{s}}(2 l+1)\right) \Omega+i \frac{L_{n}}{L_{s}}\left(\frac{1+\eta_{i}}{\tau}\right)(2 l+1)=0,\right.
$$

where $l$ is the radial eigenmode number. Two different roots of Eq. (3) can be easily identified in the simple limit where $\left|b,\left(1+\eta_{i}\right) / \tau\right| \ll 1$ and $\left|\left(L_{n} / L_{s}\right)\left(1+\eta_{i}\right) / T\right| \ll 1$; namely,

$$
\Omega \approx i \frac{L_{\mathrm{n}}}{L_{2}}(2 l+1)\left(\frac{1+\eta_{i}}{\tau}\right)
$$


and

$$
\Omega \approx 1-i(2 l+1) \frac{L_{n}}{L,}\left(\frac{1+\eta_{2}}{\tau}+1\right) .
$$

For the usual case of $\eta_{1}>0$. Eq. (4a) represents the sound wave destabilized by the ion temperature gradient (i.e., the familiar $\eta_{i}$-mode), and Eq. $(+b)$ corresponds to the shear-damped universal electron drift wave. However. when the density profile is inverted such that $\eta_{i}<0$ (throughout this paper $L_{s}, L_{T}>0$ is assumed), the situation changes. It is intuitively clear that the $\eta_{i}$-mode should be unstable for negative values of $\eta_{i}$ with $\left|\eta_{i}\right| \gg 1$. since the dependence on $L_{n}$ should be weak. ${ }^{12}$ Indeed, in the limit of $L_{n} \rightarrow \infty$. Eq. (3) becomes.

$$
\left(1+b_{3}\right)\left(\frac{\omega}{\omega_{=T i}}\right)^{2}+\left(\frac{b_{3}}{T}+i \frac{L_{T}}{L_{3}}(2 l+1)\right)\left(\frac{\omega}{\omega_{* T}}\right)+i \frac{L_{T}}{L_{3}}\left(\frac{2 l+1}{T}\right)=0 .
$$

Specifically, for $b_{s} / \tau,\left(L_{T} / L_{S}\right)(2 l+1) \ll 1$,

$$
\left(\frac{\omega}{\omega+T i}\right) \approx \pm\left(\frac{\partial l+1}{T}\right)^{1 / 2}\left(\frac{L_{T}}{L_{S}}\right)^{1 / 2} e^{\frac{-\pi}{i},}
$$

so that there exists an unstable mode regardless of the sign for $L_{n}$. As the inverted density profile peaks further, the $\left|\eta_{i}\right|$ value decreases. Then, Eqs. (4a) and ( $4 \mathrm{~b}$ ) become relevant again. Noting that $\Omega=\omega / \omega_{* e} \propto-\omega$, for $L_{n}<0$, we see that the root described by Eq. (4a) is stable for $\eta_{1}<-1$ while another root described by Eq. (4b) is unstable for $\eta_{i}<-(1+\tau)$. Therefore, for $L_{n}<0$, we should identify the root given by Eq. (4b) as an $\eta_{i}$ mode based on the physics origin of its thermodynamic driving force, despite the fact that this mode happens to be propagating in the $w_{=}$direction. Of course, Eq. (4) is an approximate solution of Eq. (3). Straightforward inspection of Eq. (3) indicates that marginal stability occurs when $\eta_{i}=-1$ at $\Omega=0$, and when $\eta_{i}=-(1+\tau)$ at $\Omega=1$. These results imply that the $\eta_{i}$-mode is unstable for $\eta_{i}>-1$ and $\eta_{i}<-(1+\tau)$. It is already clear that a threshold $a^{:} \eta_{1}=-1$ should not be accepted since the fluid approximation $\left(|\omega| \gg\left|\hbar_{\|}\right| v_{t_{1}}\right)$ breaks down badly at $\Omega=0$. On the other hand, another threshold at $\eta_{\mathrm{s}}=-(1+\tau)$ which occurs when $\Omega=1$. does not violate the fluid approximation. When $k_{\|}$ is evaluated for typical mode widths given by $E_{q}$. (2), $\left|k_{\|}\right| v_{s i} / w|\sim| L_{n} /\left.L_{s}\right|^{1 / 2}$ i.e., the fluid approximation is satisfied if $\left|L_{n}\right|<L$. 
We next proceed to analyze the kinetic eigenmode. Eq. (1), for the purpose of evaluating the threshold value of $\eta$ more accurately.

a) Normal Density Profile (Positive $\eta_{n}$-Modes); For $0<L_{n} / L$, $\ll 1$. results from a number of numerical studies ${ }^{20,21}$ have indicated that $1<\eta_{c c}<$ 2. This implies that the fluid description (which leads to $\eta_{i c}=-1$ ) cannot be used even as the lowest order approximation. Therefore, we must deal with the strong ion Landau damping region where $|\omega| \lesssim\left|k_{\|}\right| v_{t i}$. If we use the small $\xi_{1}$ (large $x$ ) asymptotic formula for the Z-function. i.e., $Z \cong i \sqrt{\pi} \xi_{1} e^{-i_{1}^{2}}$. then Eq. (1b) simplifies to

$$
Q_{\infty}(x)=\frac{\left(\tau-b_{s}\right)(\Omega T+1)-\left(\tau+b_{s}\right) \frac{\eta_{1}}{2}}{1+\frac{\eta_{t}}{2}+\Omega \tau}-\frac{i}{\sqrt{\pi}} \frac{(2 \tau)^{1 / 2}(\tau+1)}{1+\frac{\eta_{t}}{2}+\Omega \tau}\left(\frac{L_{n}}{L_{s}}\right) x
$$

Sote that the second term is small for typical values of the turning point $x$ when $L_{n} / L$, $\ll 1$. Indeed, when $L_{n} / L$, $\ll 1$, the first term of Eq. ( 7 ) alone determines the stability conditions. If we consider $\mathrm{Eq}$. (1) as a time-independent Schrödinger equation problem with the effective potential $V(x)\{\equiv-Q(x)]$, then the sign of $Q_{\infty}(x)$ determines the existence of an integrable solution. Numerical solutions indicate that $|\Omega| \ll 1$ is always satisfied at marginal stability. Hence, from the first term of $Q_{\infty}(x)$, we can see that the potential barrier is formed only when

$$
\eta_{i}>\eta_{i c}^{(+)}=\frac{2\left(\tau-b_{s}\right)}{\tau+b_{s}},
$$

and there exists an integrable solution (i.e., unstable mode). If $0<\eta_{1}<\eta_{1 c}^{i+1}$. the eigenfunction becomes unbounded in space; i.e., it is stable.

In fact, Eq. (8) is obviously just the small-b $b_{i}$-limit of the local kinetic result of Kadomtsev and Pogutse. ${ }^{23}\left(\eta_{i}^{K P}=2 /\left[1+2 b_{i}\left(1-I_{1} / J_{0}\right)\right]\right.$, where $I_{0}$. $I_{1}$ are modified Bessel functions. It is indeed expected that we can recover the local kinetic result in the $L_{n} / L, \rightarrow 0$ limit.

b) Flat Density Profile(Weak-Density-Gradinnt $\eta_{i}$-Modes): As $L_{n}$ is increased, the second term of $Q_{\infty}(I)$ in $\mathrm{Eq}$. (7) becomes larger. Furthermore, Eq. (2) indicates that the potential well at the rational stiface. 
$V_{\text {eff }}(0)=-Q(0) \simeq \frac{r}{1+\eta_{0}}$, becomes shallower. The eigenfunction accordingly extends more into the large $\mathrm{x}$ region, thereby supporting the significance of the second term of $Q_{\infty}(x)$. In this case, we can approximately solve the eigenmode equation by using WKB procedures. The appropriate quantization condition is

$$
\int_{-x_{T}}^{x_{T}} d x \sqrt{Q(x)}=(l+1 / 2) \pi,
$$

where $l$ is the radial eigenmode rumber and $x_{T}$ is the turning point in the complex plane. Noting that $x_{T}$ is determined by the balance of the first and second terms of Eq. $(\bar{i}), \boldsymbol{x}_{T} \sim \frac{L_{2}}{L_{T}} \gg 1$. On the other hand, the ion Landau damping point $x_{\mathrm{z}} \sim \Omega \frac{L_{2}}{L_{n}}$ never becomes much greater than one at marginal stability. Hence, the integral can be approximated by ignoring the getzils of $Q(x)$ for $x<x_{1}$ i i.e.,

$$
\int_{G}^{x_{T}} d x \sqrt{Q_{\infty}(x)}=(2 l+1) \frac{\pi}{4} .
$$

is mentioned earlier, $|\Omega \tau| \ll 1$ when $0<\frac{L_{n}}{L_{\mathrm{a}}} \ll 1$. When $l$ is iccreased, $|\Omega \tau|$ at marginal stability also increases. Nevertheless, $|\Omega r+1| \ll \eta_{1}$ is aiways satisfied. In the flat density limit $\left(L_{n} \rightarrow \infty\right)$, Eq. (10) yields

$$
\eta_{i c}=\frac{3}{2} \sqrt{\frac{\pi}{2}}\left(1+\frac{T_{i}}{T_{e}}\right) \frac{L_{n}}{L_{i}}(2 l+1) .
$$

The finite Larmor radius corrections bere should be ignoted in order to br consistent with the ordering $|\Omega \tau+1| \ll \eta_{i}\left[i, \Omega_{.}, b, \eta_{i}\right.$ in $E_{q} .(7)$ is also ignored in comparison to $\eta_{i}$. Equation (11) indicates that the thrsshoid condition for $\eta_{i}$ instability is independent of $L_{n}$. It also applies to negative values of $L_{n}$ provided $\left|\eta_{i}\right| \gg 1$ is satisfied. For practical applicaíions to tokamak confinement corsiderations, such as $\eta_{i}$-mode-based trarsport models, it is desirable to have an expression for $\eta_{i c}$ which is valid for arbitrary values of $L_{n} / L_{\text {s. }}$ In fact, if we can ignore the ' $\Omega \tau$ ' term in Eq. (7), then Eq. (10) yiejds the following expression:

$$
\left(1+b_{1}\right)^{3}\left(\eta_{i c}-\eta_{t c}^{(1)}\right)^{3}=\frac{9 \pi}{8}\left(1+\frac{T_{t}}{T_{e}}\right)^{2}\left(\frac{L_{n}}{L_{s}}\right)^{2}(2 l+I)^{2}\left(\eta_{\mathrm{tc}}+2\right)
$$


As mentioned earlier, the finite-gyroradius corrections should be ignosed for $L_{n} / L_{3} \geq 1$, in order to be consistent with the ordering $|\Omega \tau+1| \ll \eta_{1}$. This can be done by replacing $\left(1+b_{i}\right)^{3}$ with 1 , sirce the details of $\eta_{x c}^{(+1}$ do not affect the answer in the weak density gradient limit $\left(L_{n} / L, \geq 1\right)$.

The resultant form of the algebraic equation. Eq. (12) (with $1+b$, replaced by 1). can be readily solved exactly to yield:

$$
\eta_{\mathrm{kc}}=\eta_{\mathrm{sc}}^{(+)}+\sqrt[3]{b+\sqrt{b^{2}-a^{3}}}+\sqrt[3]{b-\sqrt{b^{2}-a^{3}}} .
$$

where

$$
\begin{gathered}
\eta_{1 c}^{(+)}=2\left(1-b_{i}\right) /\left(1+b_{i}\right) \\
a=\frac{3 \pi}{8}\left(1+\frac{T_{i}}{T_{e}}\right)^{2}\left(\frac{L_{n}}{L_{t}}\right)^{2}(2 l+1)^{2} \\
b=3\left(1+\frac{1}{2} \eta_{i c}^{(+)}\right) a .
\end{gathered}
$$

When $0<L_{n} / L$, $<1 . b^{2} \gg a^{3}$, and the second tarm on the tight side of $\mathrm{Eq}$. (13) provides the nonlocal correction to $r_{/ i c}^{(+)}$. The third term on the right side of Eq. (13) is negligible in this case. When $L_{n} / L$, becomes large enough so that $b^{2}<a^{3}$, the second and third terms of $\mathrm{Eq}$. (13) become complex. However. since they are complex-conjugates to each other, $\eta_{i c}$ remains a real value. In this case, the following equivalent formula minimizes the possibility of confusion in choosing the right complex root:

$$
\eta_{\mathrm{sc}}=\eta_{\mathrm{xc}}^{(+)}+\sqrt{\frac{3 \pi}{2}}\left(1+\frac{T_{1}}{T_{\mathrm{t}}}\right) \frac{L_{\mathrm{n}}}{L_{\mathrm{s}}}(2 l+1) \cos \left[\frac{1}{3} \cos ^{-1}\left(\frac{b}{a^{3 / 2}}\right)\right] .
$$

If $L_{n} / L, \gg 1$. the $a^{3}$ term dominates the right side of Eq. (13). and we thereby recover Eq. (11).

Before proceeding to the analysis of negative $\eta$, modes, it is appropriale here to compare our threshold resu'i for the flat density gradient limit. Eq. (11), with recent estimates ${ }^{13-16}$ for the onset of that density gradient toroidal $\eta_{s}$ instabilities. In terms of the critical ion temperature gradient scale length $\left(L_{T i}\right\}$, Eq. (11) can be rewritten as

$$
\left(\epsilon_{L_{T}}\right)_{c}=\frac{2}{3} \sqrt{\frac{2}{\pi}} \frac{L g}{R} /\left(1+\frac{T_{1}}{T_{e}}\right)
$$


With $c_{L_{T}} \equiv L_{T_{0}} / R . L,=R_{q} / \dot{s}$, and $\dot{s}=r q^{\prime} / q$ Romanelli has reported that when ion $\Gamma B$-drift and curvature drift resonant effects are taken into account. the threshold for toroidal $\eta_{1}$-modes in the weak density gradient limir can be approximated by

$$
\left(\epsilon_{L_{T_{1}}}\right)_{\mathrm{c}}=\frac{3}{4} /\left(1+\frac{T_{1}}{T_{\mathrm{e}}}\right)
$$

The absence of stear $(L$,$) dependence in \mathrm{Eq} .(15)$ is due to the fact that the ion transit motion is neglected. Comparing the sheared-slab and toroidal results one can see that for values of $L_{*} / R$ around 1.4, Eqs. (14) and (15) are in agreernent. If $T_{t}=T_{e}$, then Eqs. (14) and (15) yield $\left(\epsilon_{t_{*}}\right)_{c} \simeq 0.375$. This value. in turn, is consistent with more recent toroidal studies ${ }^{15.16}$ which have indicated the flat gradient threshold to be $\left(t_{L_{\tau_{2}}}\right)_{c} \simeq 0.35$ under similar conditions. Hence. in the limit of weak density gradients, the present shearedslab result Eq. (14) and the approximate toroidal kinetic results ${ }^{15.16}$ provide similar estimates for the critical $L_{T}$.

c) Inverted Density Profile (Negative $\eta_{\mathrm{r}}$-Modes): is discussed in the beginning of this section the threshold for inverted density profile is

$$
\eta_{\mathrm{ic}}^{(-1}=-\left(1+\frac{T_{e}}{T_{i}}\right)
$$

This equation has been derived from the dispersion relation. Eq. (15), and agrees very well with the numerical results obtained from the WKB and shooting code analyses of the kinetic eigenmode equation, Eq. (1). Figure 1 illustrates the excellent agreement between Eq. (16) and the numerical results for various values of $T_{e} / T_{1}$. Finite Larmor radius corrections to Eq. (16) are negligible for the long wavelength modes considered in this paper $\left(b_{1} \leqslant 0.25\right)$. A particularly interesting new feature for the inverted density profile cases is that $\gamma \ll\left|\omega_{\gamma}\right|$ is satisfied for $\eta_{i}$ modes over a wide range of negative $\eta_{1}$ values. This is demonstrated in Fig. 2 where the complex $\Omega$ values obtained from the numerical analysis are plotled as functions of $\eta_{1}$ for fixed values of $L_{n} / L_{3}=-1, b_{1}=0.1$, and $\tau=1$. This differs considerably from the positive $\eta_{\mathrm{t}}$ case where $\left.\gamma \ll \mid w_{r}\right]$ is satisfied only near the threshold. $\eta_{i c}^{[+]}$. The comparison between negative and positive $\eta_{0}$ cases is made in Figs. $3 a$ and $3 \mathrm{~b}$ where $\eta_{\mathrm{t}}$ is varied for fixed $L_{T} / L_{S}=0.05, b_{i}=0,1$, and $T=1$. With 
regard to the weak density gradient limit, Fig. $3 c$ shows that. as expected. the complex eigenfrequencies are almost symmetric with respect to the sign of $L_{n}$, when $\left|\eta_{i}\right| \gg 1$ is satisfied.

The threshold conditions for various values of profile scale lengths ( $L_{T}, L_{n}$. and $\left.L_{3}\right)$ are numerically calculated and compared to the analytic formulas. Eqs. (8). (11), and (16). As illustrated in Fig. 4a, a very good agreement is found with the exception of cases where $L_{n} / L, \simeq-1$. This is not surprising because neither the fluid approximation used for the negative $\eta_{i}$ analysis nor the upproximate kinetic approach employed in the flat density profile calculations is justified in this limit. Note that the change in mode character is rather abrupt as a function of $L_{n} / L_{*}$ in this region. This transition region is shown in detail in Fig. 4b. Additional numerical results for normal and flat density profiles and their comparisons with the theoretical formulas. Eqs. (\$) and (13), are presented in Figs. 5, 6, and 7. These include both poloinal and radial wave number dependences as well as the temperature ratio dependencies. The implications of these results for tokamak confinement physics are discussed in the next section.

\section{ROLE OF $\eta_{i}$-INSTABILITIES IN H-MODE PLAS- MAS}

In this section, we discuss the results preserted in Sec. II and their relevance to tokamak experiments. We begin with the positive $\eta_{7}$ cases for which Eq. (13) is applicable. Sperifically, for a sharply peaked density profile and low magnetic shear, $L_{n} / L_{s} \ll 1$, so that $\eta_{i c} \cong 2\left(1-b_{i}\right) /\left(1+b_{1}\right)$. This indicates the shorter wavelength modes can be easily excited. This property is also illustrated in Fig. 5 . However, for the associated larger values of $b_{i}$ (i.e., $\left.b_{1} \sim 1\right) \rho_{i}^{2} k_{x}^{2}$ also tends to be large. Hence, an integral equation formulation is necessary to analyze the stability of these modes in detail. ${ }^{23.24}$

When $L_{n} / L$, becomes non-negligible either due to fattcr density profilas or to stronger magnetic shear, we must consider the full expression for $\eta_{\mathrm{x}}$ given by Eq. (13). In this case, $\eta_{\text {i }}$ modes become more difficult to excite if $T_{z} / T_{e}$ is rajsed. This dependence is rather strong and may suggest $t$ reconciliation of high $T_{1}$ and the relatively benign fuctuations and transport observed in the PLT neutral-beam-heated plasmas. ${ }^{25}$ This possible link between a $T$ dependent $\eta_{1}$-threshold and ihe PLT result was first suggested by S. Guo et 
al. ${ }^{26}$ In Ref. 26 the Savorable $T_{i} / T_{e}$ aependencs of $\eta_{1 c}$ was pradicted only for the relatively short wavelength modes where $b_{s} \equiv T / 2$. Hence, the differertial equation used ${ }^{26}$ is only marginally valid. On the other hand, ou present result [Eq. (13)] indicates that the favorable $T_{i} / T_{\varepsilon}$ depenoence persists even tor long wavelength inodes $\left(b_{i} \ll 1\right)$ when values of $I_{n} / L_{\text {s }}$ are fini:3. Figure 6 illustrates the strong $T_{i} / T_{e}$ dependence for $L_{n} / L_{s}=1$ and $L_{n} / i_{n}=0.1$. This favorable, sependence of the stability threshold on $T_{1} / T_{e}$ should persisi even in the hot ion mode of TFTR operation. In the T'FTR cases. however, the non-Maxwellian ion beam contribution c.in be significant. ${ }^{27}$ Hence, Eq. (13), which was derived assuming a $M$ rwellian distribution for the ions should only be regarded as being indicative of a quantitative physics trend for these situations. Examination of Eq. (13) also indicates that the higher radial eigenmodes should have nigher instability thresholds. The difference in the threshold values is small when $\left(1+T_{i} / T_{e}\right) L_{n} / L, \ll 1$. However, when $\left(1+T_{i} / T_{e}\right) L_{n} / L_{5}$ becomes non-uegilgible, the difference becomes larger and indicates that these higher radial eigenmodes are harder to excite linearly. These facts are illustrated in Fig. 7. The linear stability properties of such higher radial eigenmoces suggest that they can become significant ${ }^{2 \mathrm{~g}}$ in cases where the shear is relatively weak and the density frofile is sather peaked. As mentioned in Sec. I, recent measurements in DIII-D H-mode plasmas have indicated that the characteristic electron density profiles can be nearly flat and sometimes even inverted (outwardly peaked), ${ }^{10,17}$ Under these circumstances either Eq. (11) for the flat density profile cases or Eq. (16) for the negaiive $\eta_{i}$ situation s'iould be applicable. However, it is difficult to derive a formula which covers both types of conditions. This is because Eq. (16) has been derived from the luid eigenmode equation where $|\omega| \gg\left|k_{\|}\right| v_{t_{1}}$ is assumed, while the asymptotic behavior in the region where $|\omega| \ll\left|k_{\|}\right| v_{t i}$ plays a crucial role in the derivation of Eq. (11). It is worthwhile to remark that in contrast to Eq. (11), Eq. (16) predicts a higher threshold $\left(\left|\eta_{i c}^{(-)}\right|\right)$ for larger $T_{e} / T_{i}$ when the density profile is inverted. Also, to the dominant order shown in Eq. (11), the threshold is independent of poloidal and radial wavelengths. This fact has been confirmed from numerical studies using a WKB code and a shooting code. We also note that for flat density profiles the poloidal wave number dependence obtained from our numerical studies is weak.

Rough estirrates based on typical scale lengths of profiles obcained from 
DIII-D H-mode discharges ${ }^{16,29}$ suggest values of $\eta_{i}$ which exceed the thresholds given by Eq. (11) and Eq. (16). For example, using the $T_{i}(r)$ and $n_{i}(r)$ profiles for $0.4 \leq r / a \leq 0.5$ from Ref. 29 , we obtain $L_{T i} / R \cong 0.19$, $L_{N i} / R \cong-1.18$ which yield $\eta_{i}=-6.21$. As mentioned in the previous section, the negative $\eta_{i}$ modes which are likely to be excited in various $\mathrm{H}$-mode discharges are chatacterized by $\gamma \ll\left|\omega_{R}\right|$. This suggests that a weak turbulence theory of these instabilities is needed to gain further insight into their influence on ic $n$ thermal transport. For the weak density gradient limit, the $T_{i}(r)$ profiles should stay reasonably close to the marginal stability condition in order to be consistent with the observed confinement properties. This again suggests that a weak-turbulence-type nonlinear analysis of the associated transport coefficient is appropriate. Investigations in this direction for the negative $\eta_{i}$ situations are in progress, and the results will soon be presented in another paper. An encouraging feature of this analy'sis is that it is possible to treat the 'test' mode using fluid equations even for ralues of $\eta_{i}$ near $\eta_{i c}$. This makes feasible a nonlinear nonlocal analysis, where the actual radial structure of the eigenmodes is properly taken into account. With regard to other weak-turbulence-type studies, we note that a local kinetic analysis of positive $\eta_{\mathrm{i}}$-modes near threshold has recently been presented by Mattor and Diamond. ${ }^{30}$ Regarding the radial mode structure, the crucial difference between positive $\eta_{i}$ mode and negative $\eta_{i}$ mode is that the kinetic analysis is essential for the former case while the fluid approximation is valid for the latter. This contrast is demonstrated in Fig. 8.

A preliminary iterative evaluation of the fluctuation spectrum for margizally unstable negative $\eta_{i}$-modes has indicated that the weak turbulence form of the ion diffusivity is considerably smaller than the familiar mixing length estimate.

\section{SUMMARY}

Motivated by experimental results for the profile characteristics associated with $\mathrm{H}$-mode plasmas, we have carried out a systematic analytical and numerical study of the linear properties of $\eta_{i}$-instabilities in a sheared slab geometry. Ion transit resonance effects, which can strongly influence the stability thresholds, have been taken into account in our investigation of normal $\left(\eta_{i}>0\right)$, flat $\left(\eta_{i} \rightarrow \infty\right)$, and inverted $\left(\eta_{i}<0\right)$ ion density profile sce- 
narios. The corresponding analytic threshold criteria given, respectively, by Eqs. (8), (11), and (16) are well supported by utumerical results from WKB and Shooting Code programs. With regard to the weak-density-gradient $\eta_{i}$ modes, Eq. (II) is a new result for the ion temperature gradient threshold when ion transit resonances are included. As noted in the discussions following Eqs. (14) and (15), the present criterion from our sheared-slab analysis compares reasonably well with estimates for the critical $L_{T i}$ from recent investigations of weak density gradient toroidal $\eta_{i}$-modes.

Equation (16) and the associated numerical results presented in Sec. II represent the first derivation of threshold conditions for negative $\eta_{i}$-modes along with the properties of these instabilities when the thresholds are exceeded. Of particulat significance is the fact that the growth rate is much smaller than the real frequency, even far away from the threshold for these negative $\eta_{i}$-modes. This trend tends to hold true even when toroidal modifications such as trapped particle dynamics are iatroduced. For example, in earlier work un trapped-particle drift modes by Tang et al. ${ }^{31}$ it was shown that the negative $\eta_{i}$ form of trapped-ion modes was clearly weaker than its positive $\eta_{i}$ counterpart. Moreover, the property $|\gamma| \omega_{A} \mid \ll 1$, derived in the present work, is also characteristic of the negative $\eta_{i}$ trapped-ion instabilities.

In general, the feature, $\left|\gamma / \omega_{R}\right| \ll 1$, helps to justify the applicaticis of the standard weak turbulence procedure ${ }^{32}$ for calculating the transport associated with the resence of negative $\eta_{i}$ modes. The primary physics elements of this nonlinear analysis and their implications for $\mathrm{H}$-mode confinement have been qualitatively discussed in Sec. III. In addition, it should be noted that the stabilizing influence of finite beta effects ${ }^{24}$ on the shorter wavelength $\eta_{i}$ modes can significantly influence the nonlinear results. Specific results for transport along with quantitative details of the analysis will soon be presented elsewhere. The major intent of the present paper bas been to establish clearly the linear properties of $\eta_{i}$-modes with special attention focused on the negative ion density gradient scenario common to many steady-state $\mathrm{H}$-mode plasmas. ${ }^{[1,17}$

\section{ACKNOWLEDGEMENTS}

The authors are grateful to Dr. R. B. White for providing the WhB and Shooting Code programs used to carry out the numerical computations 
in the present paper and Mr. Y. J. Chung for providing Fig. 7. This work is supported by U.S. Department of Energy Contract Vo. DE-AC02-76CH03073. 


\section{References}

${ }^{1}$ B. Coppi, M. N. Rosenbluth, and R. Z. Sagdeev, Phys. Fluids 10, 382 (1967).

${ }^{2}$ W. Horton, D-T. Choi, and W. M. Tang, Phys. Fluids 24, 1077 (1981).

${ }^{3}$ G. S. Lee and P. H. Diamond, Phys. Fluids 29, 3291 (1986).

${ }^{4}$ F. Romanelli, W. M. Tang, and R. B. White, Nucl. Fusion 26, 1515 (1986).

${ }^{5}$ R. R. Dominguez and R. E. Waitz, Nucl. Fusion 27, 65 (1987).

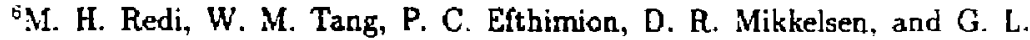
Schmidt, Nucl. Fusion 27, 200 (1987).

'M. H. Redi, W. M. Tang, D. K. Owens, M. Greenwald, O. Gruber. and M. Kaufinann, Princeton Plasma Physics Laboratory Report PPPL-2525, 1988 (submitted to Nucl. Fusion).

${ }^{8}$ D. L. Brower, W. A. Peebles, S. K. Kim, N. C. Luhmann, Jr., W. M. Tang, and P. E. Phillips, Phys. Rew. Lett. 59, 49 (1987).

${ }^{9}$ D. L. Brower, S. K. Kim, W. M. Tang, M. H. Redi, M. E. Austin, R. B. Bengston, R. V. Bravenec, S. P. Fan, M. S. Foster, R. F. Gandy, Y. X. He, N. C. Luhmann, Jr., S. C. McCool, A. Ourous, D. M. Patterson, W. A. Peebles, P. E. Phillips, T. L. Rhodes, B. Richards, C. P. Ritz, W. L. Rowan, and T. L. Wooton, "Experimental Observation of Ion-Temperature-Gradient-Driven Turbulence in the TEXT Tokamak," in Proc. of 15th European Conf. on Cont. Fusion and Plasma Heating (European Physical Society, Dubrovnik, 1988), Vol. 12B, Part 1, p. 183.

${ }^{10}$ K. H. Burrell, S. Ejima, D. P. Schissel, N. H. Brooks, R. W. Callis, T. Carlstrom, A. P. Colleraine, J. C. DeBoo, H. Fukumoto, R. J. Groebner, D. N. Hill, H.-M. Hong, N. Hosogane, G. L. Jackson, G. L. Jahns, G. Janeschitz, A. G. Kellman, J. Kim, L. Lao, P. Lee, J. Lohr, J. L. Luxon, M. Ali Mahdavi, C. P. Moeller, N. Ohyabu, T. H. Osborne, D. Overskei, P. I. Petersen, T. W. Petrie, J. C. Phillips, R. Prater, J. T. Scoville, R. P. Seravdarian, M. Shimada, B. W. Sleaford, R. T. Snider, R. D. Stambaugh, 
R. D. Stav, H. St. John, R. E. Stockdale, E. J. Strait, T. S. Taylor, J. F. Tooker, and S. Yamaguchi, Phys. Rev. Lett. 59, 1432 (1987!.

${ }^{11}$ C. Gowers, D. Bartlett, A. Boileau, S. Corti, A. Edwards, N. Gottardi, K. Hirsch, M. Keilhacker, E. Lazzaro, P. Morgan, P. Nielsen, J. O'Rourke, H. Salzmann, P. Smeulders, A. Tanga, M. von Hellermann, "Profile Behavior During Land H Phases of JET Discharges," in Proc. of $15 t h$ European Conf. an Cont. Fusion and Plasma Healing, (European Physical Society, Dubrovnik, 1988) Vol. 12B, Part 1, p. 2239.

${ }^{12}$ W. M. Tang, G. Rewoldt, and L. Chen, Phys. Fluids 29, 3715 (1987).

${ }^{13} \mathrm{~F}$. Romanelli, "Ion Temperature Gradient Driven Modes and Anomalous Ion Transport in Tokamaks," JET - IR(87)16(1987) (submitted to Phys. Fluids).

${ }^{14}$ R. R. Dominguez and R. E. Waltz, "Ion Temperature Gradient Mode in the Weak Density Limit," General Atomics Report GA-A19032, 1987.

${ }^{15} \mathrm{H}$. Biglari and P. H. Diamond, presented at Sherwood Theory Confererce. Gatlinburg, Tennessee, 1988 (suimitted to Phys. Fluids).

${ }^{16} \mathrm{R}$. R. Dominguez and M. N. Rosenbluth, Private Communications.

${ }^{17 D}$. P. Schissel, R. E. Stockdale, H. St. John, and W. M. Tang, "Measurements and Implications of $Z_{\text {eff }}$ Profiles on the DIII-D Tokamak, "General Atomics Report GA-A199049, 1988; Phys. Fluids (in press).

${ }^{18} \mathrm{~L}$. Chen, Private Communications.

${ }^{19}$ W. M. Tang, Nucl. Fusion 18, 1039 (1978).

${ }^{20}$ R. E. Waltz, W. Pfeiffer, and R. R. Dominguez, Nucl. Fusion 20, 43 (1980).

${ }^{21}$ W. M. Tang, R. B. White, and P. N. Guzdar, Phys. Fluids 23, 167 (1980).

${ }^{22}$ B. B. Kadomtsev and O. P. Pogutse, in Review of Plasma Physics, edited by M. A. Leontovich, (Consultants Bureau, New York, 1970), Vol. 5, p. 249.

${ }^{23}$ W. M. Tang, G. Rewoldt, and E. A. Frieman, Phys. Fluids 23, 2454 (1980). 
${ }^{24} J$. Q. Dong, P. N. Guzdar, and Y. C. Lee, Phys. Fluids 30, 2694 (1987).

${ }^{25}$ R. J. Goldston and the PLT and Neutral Beam Groups, in Heating in Toroidal Plasmas, Proc. of the 2nd Joint Grenoble-Varenna Int. Symposium Como(CEC, Brussels, 1980), Vol. II, p. 711.

${ }^{26} \mathrm{~S}$. Guo, J. Shen, L. Chen, and S. T. Tsai, Acta Physica Sinica 31, 17 (1982).

${ }^{2 \pi}$ T. S. Habm, Bull. Am. Phys. Soc. 32, 1889 (1987).

${ }^{28}$ P. W. Terry J. N. Leboeuf, P. H. Diamond, D. R. Thayer, J. E. Sedlak, and G. S. Lee, Instituie for Fusion Studies Report IFSR-308, 1988 (submitted to Phys. Fluids).

${ }^{29}$ D. P. Schissel, Private Communications.

${ }^{30}$ N. Mattor and P. H. Diamond, presented at Sherwood Theory Conference, Gatlinburg, TN, 1988, Abstract $2 \mathrm{C} 9$.

${ }^{31}$ W. M. Tang, J. C. Adam, and D. W. Rass, Phys. Fluids 20, 430 (1977).

${ }^{32}$ R. Z. Sagdeev and A. A. Galeev, Nonlinear Plasma Theory, edited by T. M. O'Neil and D. L. Book (Benjamin, New York, 1969). 


\section{Figures}

FIG. 1. Plot of $\eta_{i c}^{(-)}$versus $T_{c} / T_{i}$ for $b_{s}=0.1, L_{n} / L_{\mathrm{a}}=-0.1$. The solid line and dots correspond, respectively, to the analytic Eq. (16) and to the numerical results.

FIG. 2. Plot of numerically computed $\Omega$ versus $\eta_{i}$ for $L_{n} / L_{3}=-1, b_{i}=$ 0.1 . and $\tau=1$. The solid and dashed lines correspond, respectively, to $\operatorname{Im}(-\Omega)$ and $\operatorname{Re}(-\Omega)$. Note that $\Omega=\omega / \omega_{\text {ee }} \propto-\omega$, since $L_{n}<0$.

FIG. 3. Plot of numerically computed $\Omega$ versus $\eta_{i}$ for $L_{T} / L_{S}=0.05, b_{i}=0.1$, and $\tau=1$. The black and white dots correspond, respectively, to the imaginary and to the real part of the eigenvalues. Figure 3a deals with negative $\eta_{i}$-modes, Fig. $3 \mathrm{~b}$ deals with the familiar positive $\eta_{i}$-modes, and Fig. $3 c$ deals with the weak density gradient limit of both classes of $\eta_{i-}$ instabilitjes.

FIG. 4. Stability diagram in $L, / L_{n}$ versus $L_{s} / L_{T}$ space, for $b_{i}=0.1, \tau=1$. Dots correspond to numerical results. The lines $a, b$, and $c$ correspond to analytic results obtained from Eq. (8), Eq. (11), and Eq. (16). Figure $4 b$ is a blowup of Fig. $4 \mathrm{a}$ for the weak density gradient regime.

FIG. 5. Plot of $\eta_{i c}$ versus $b_{i}$ for $L_{n} / L_{0}=0.1, \tau=1$. The dots and the line correspond, respectively, to numerical and analytical Eq- (8) results.

FIG. 6. Plot of $\eta_{i c}$ versus $T_{i} / T_{e}$ for $b_{i}=0.1$. The lower and upper curves correspond, respectively, to the numerical results for $L_{n} / L_{2}=0.1$ and $L_{n} / L_{s}=1$.

FIG. 7. Plot of $\eta_{i c}$ versus $L_{n} / L_{a}$ for $b_{i}=0.1, \tau=1$. The black dots are numerical results for the fundamental $(l=0)$ modes. The solid lines correspond to the analytical results (Eq. 13), for $l=0,1$, and 2 .

FIG. 8. Plot of radial eigenmode structures for $L_{T} / L_{9}=0.05, b_{i}=0.1$, $\tau=1$ with the solid and dashed lines denoting the real and imaginary parts of $\varphi(x)$. Ion Landau damping points $x_{i}$ where $|\omega|=\left|k_{\|}\right| v_{t i}$ are indicated. Figure $8 \mathrm{a}$ corresponds to $\eta_{i}=4$ and shows that since $\vartheta(x)$ extends considerably beyond $x_{i}$, a kinetic analysis is necessary. Figure $8 b$ 
corresponds to $\eta_{i}=-4$ and shows that since $\varphi(x)$ is localized within $x_{i}$, the fluid approach for such negative $\eta_{i}$ cases is justified. 


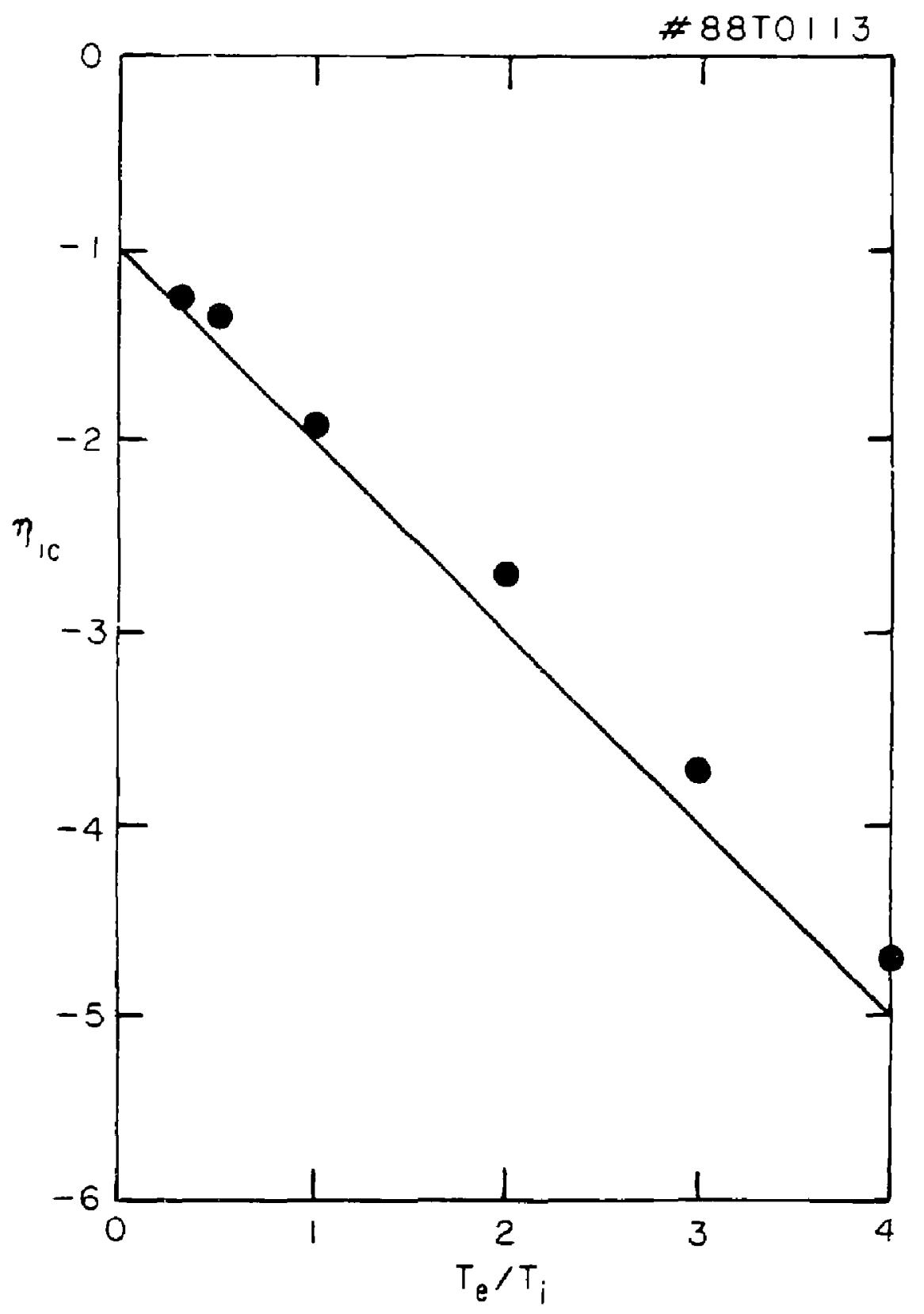

FIG. 1 


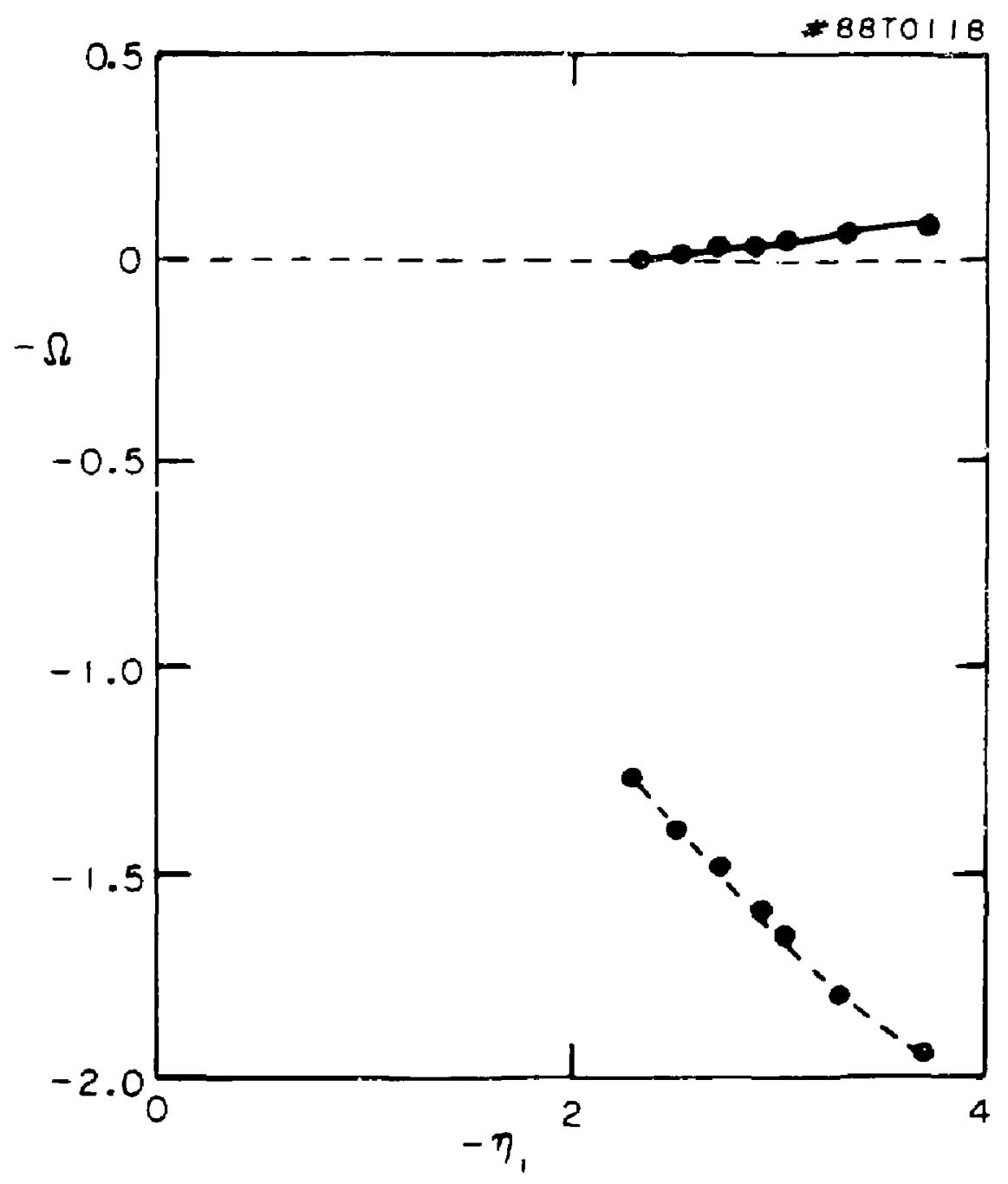

EIG. 2 


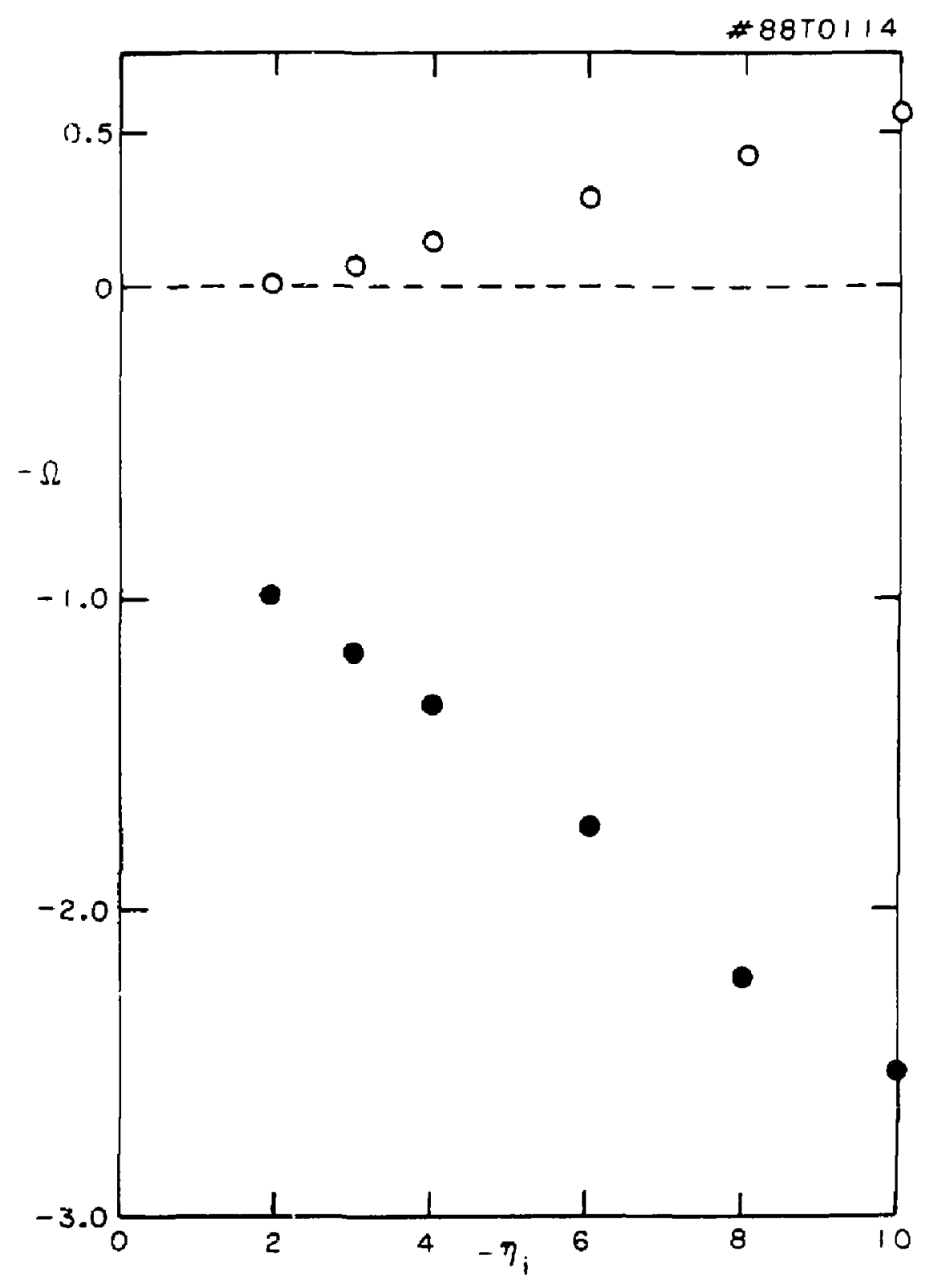

FIG. 3(a) 


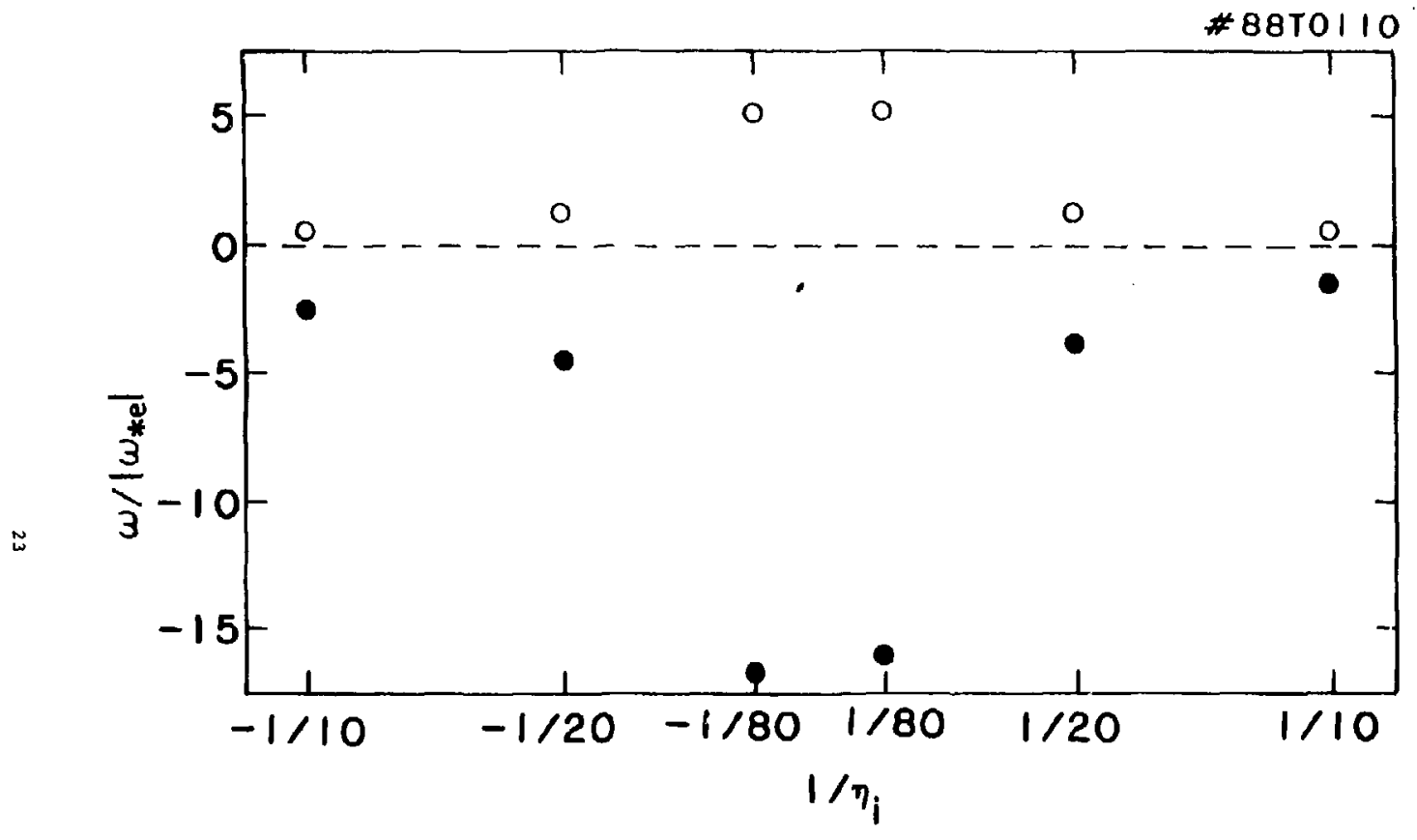




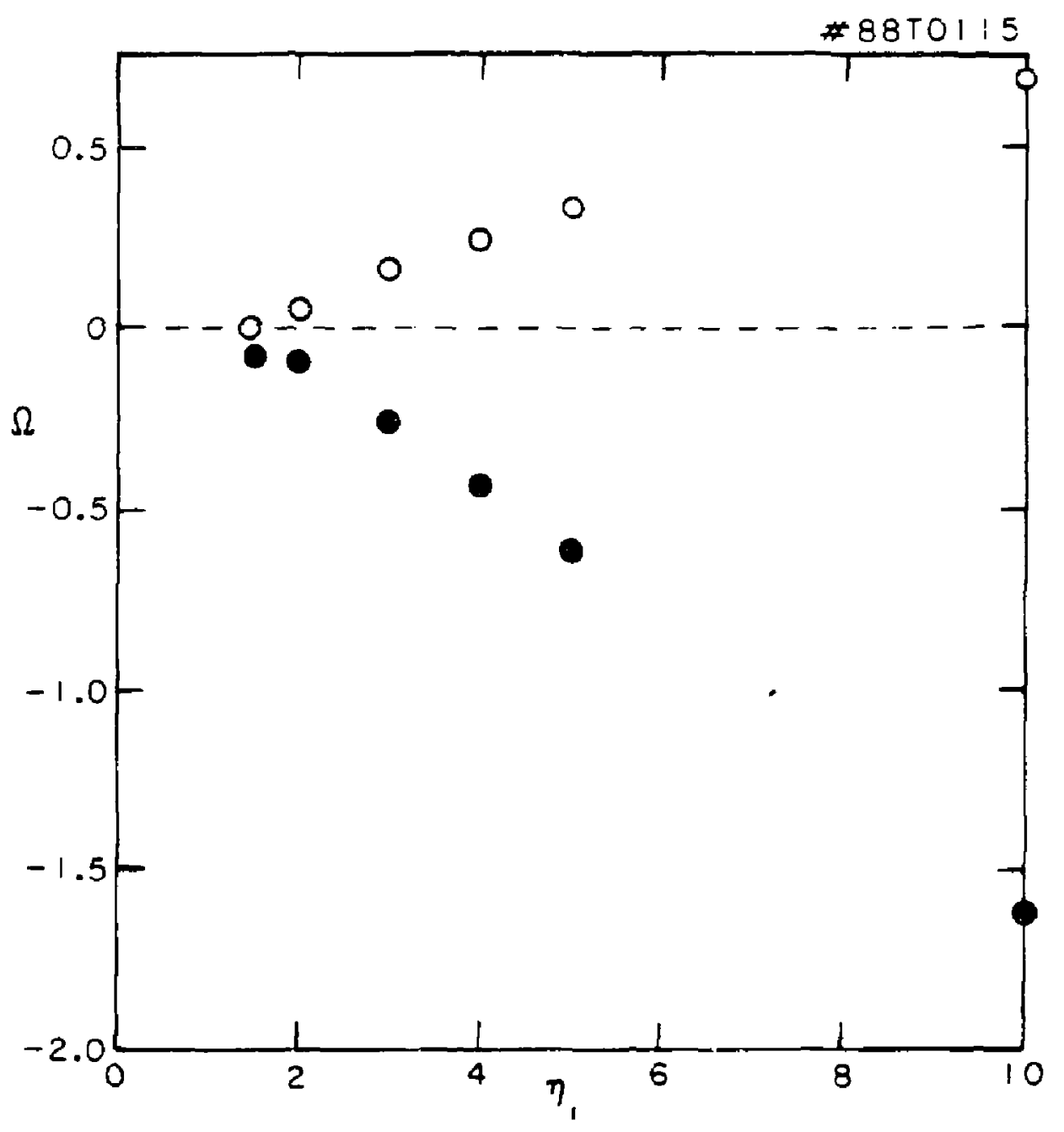

FIG, 3 (c) 


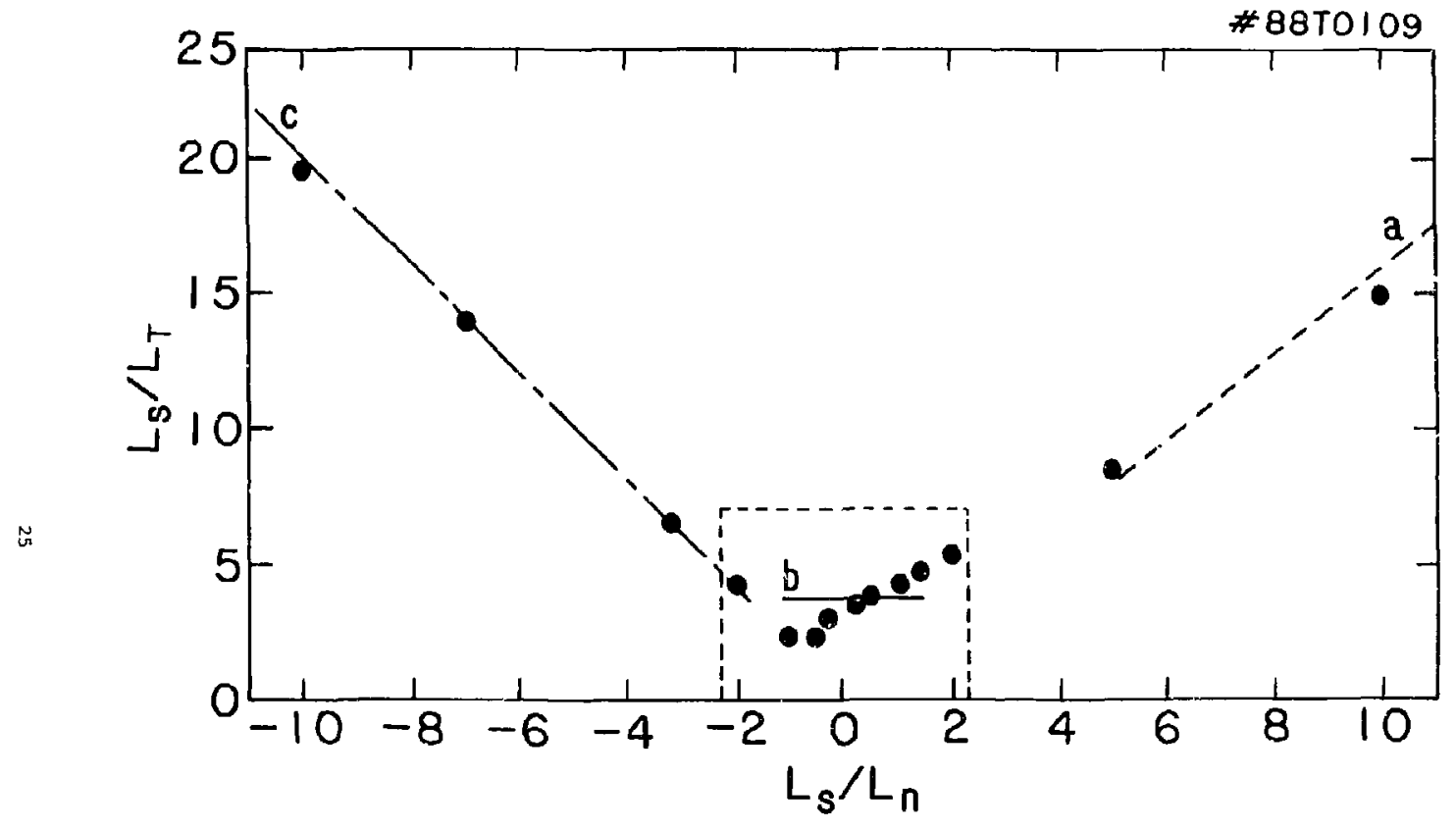

FIG. 4(a) 
\#88T01 12

ณ

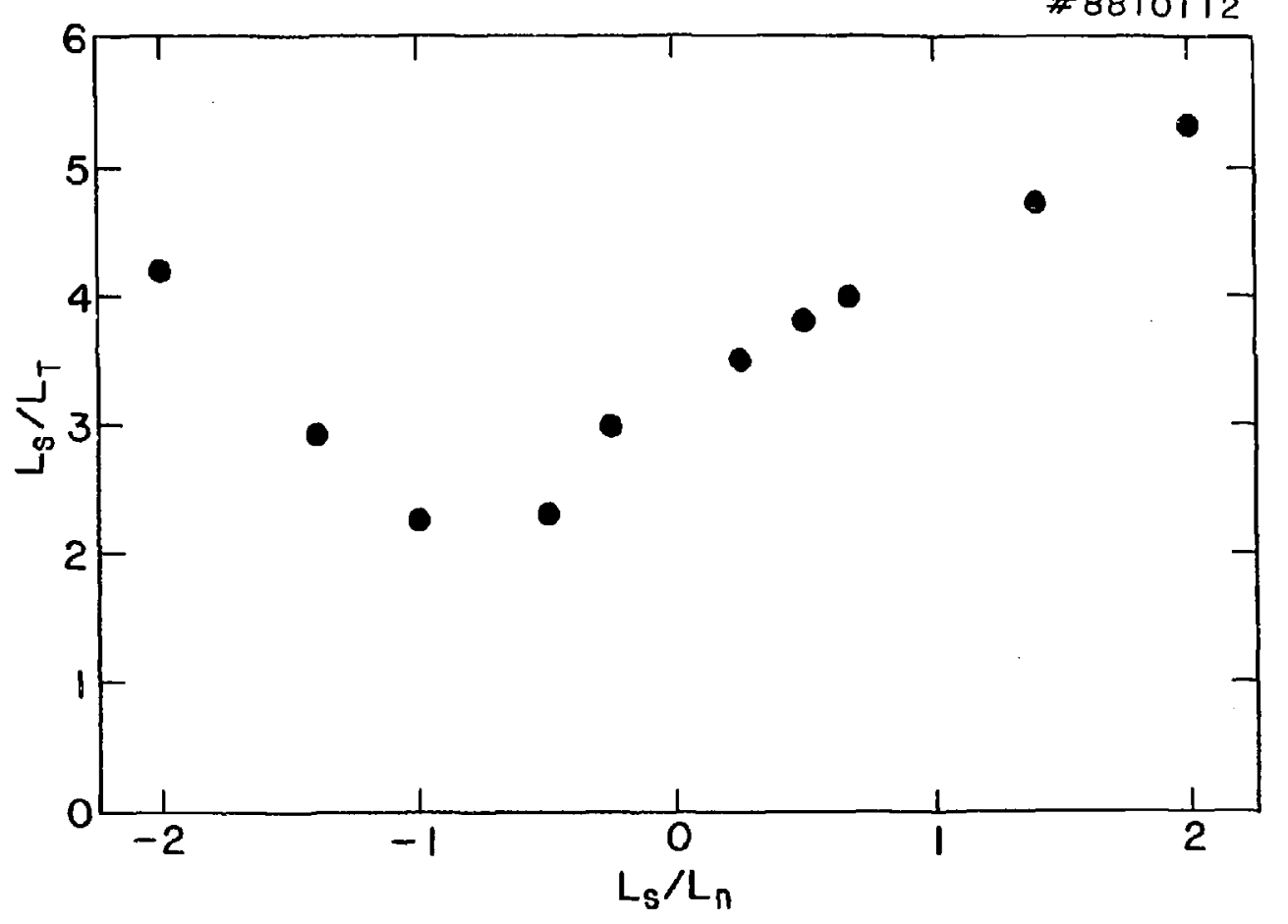

F16. $4(b)$ 


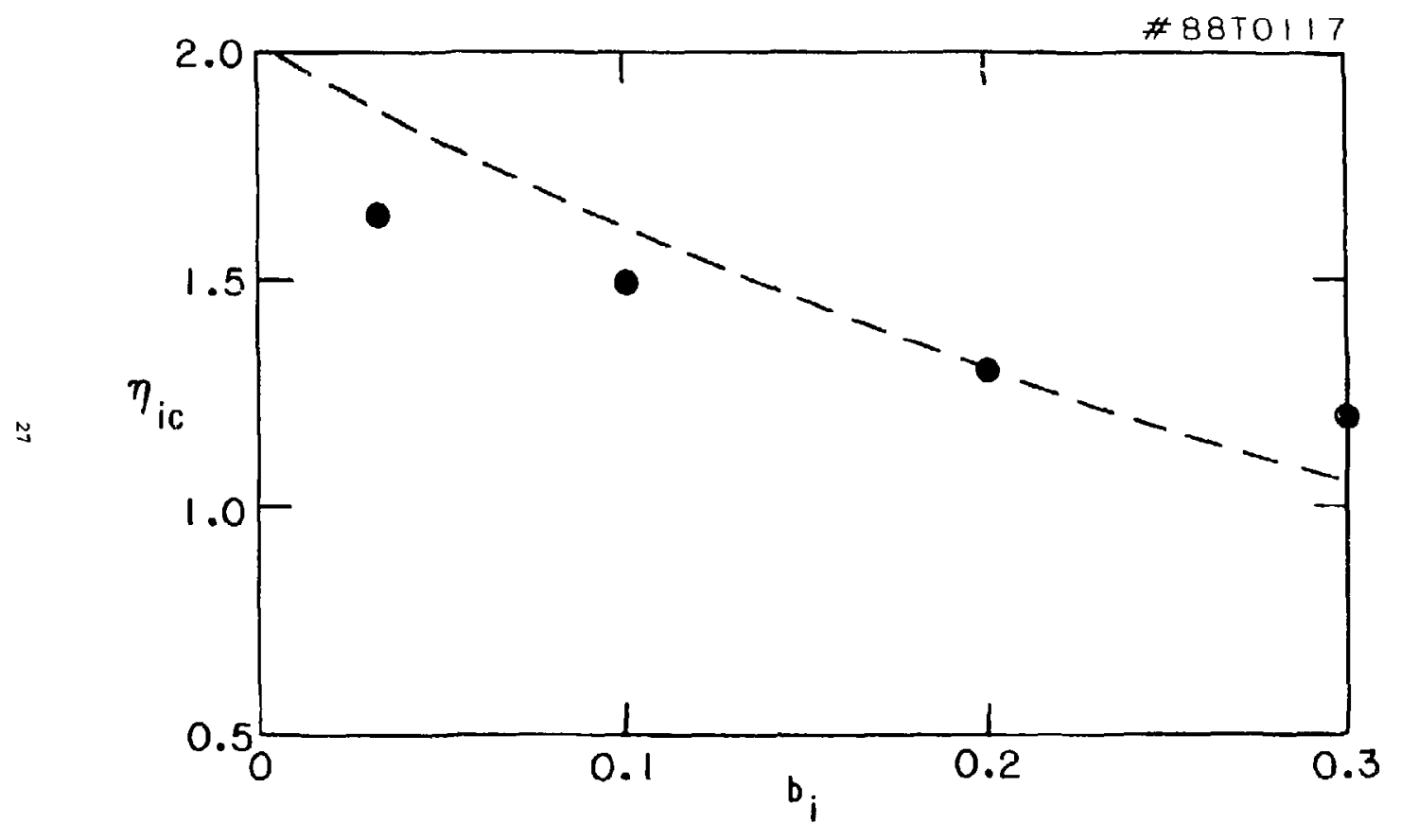

FIC. 5 


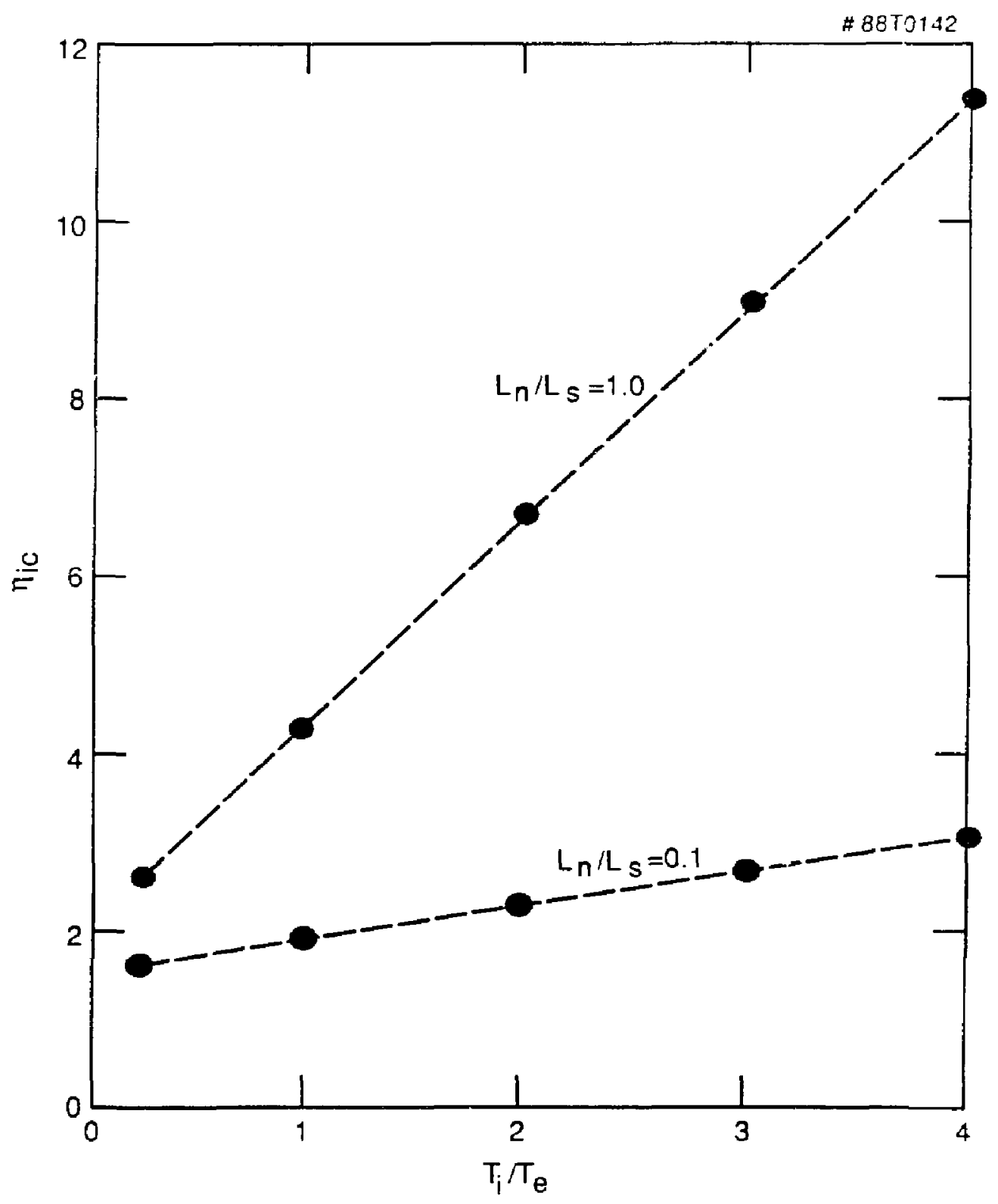

FIG. 6 


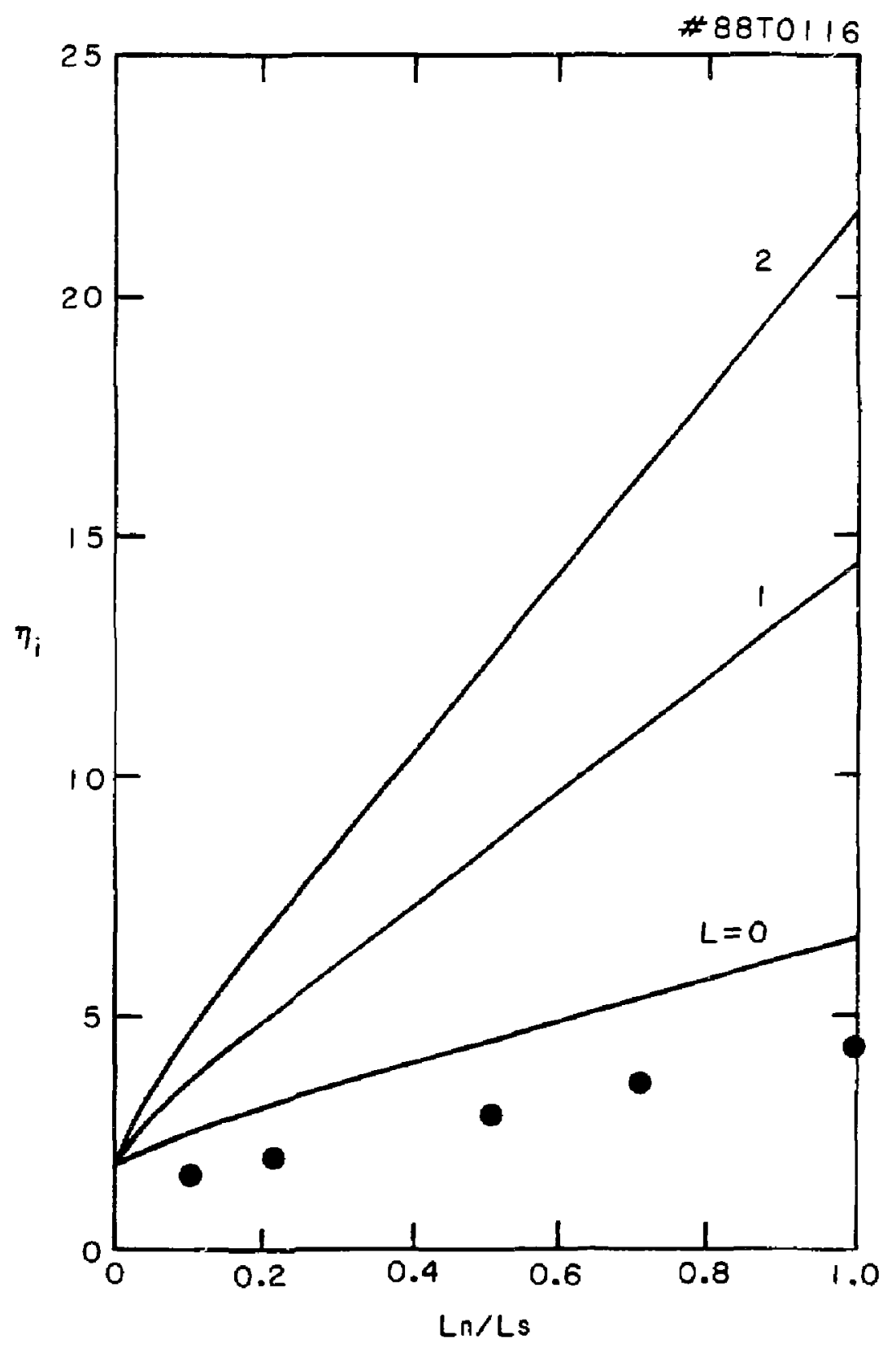

FIG. ? 


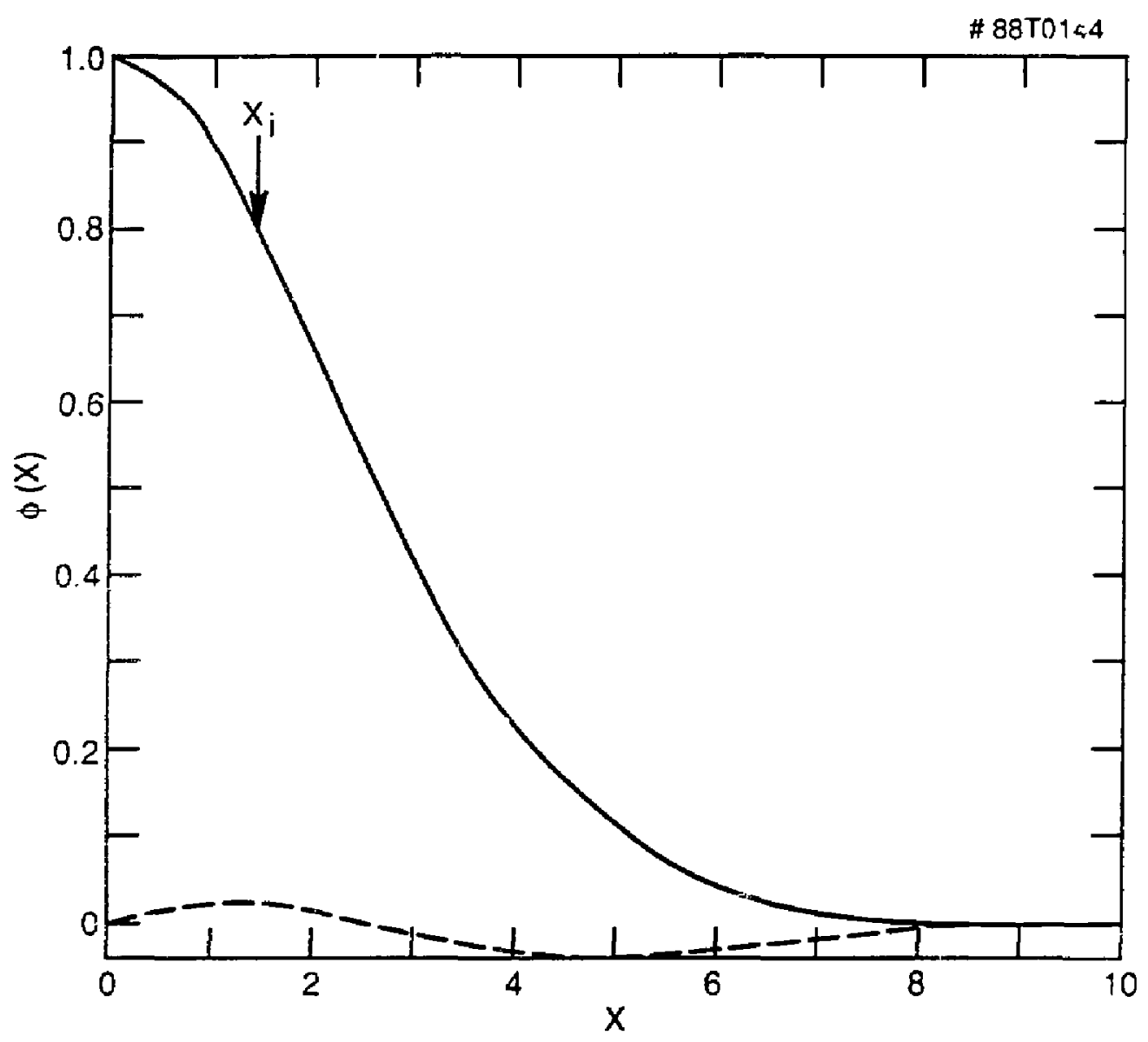

FIG. $8(3)$ 


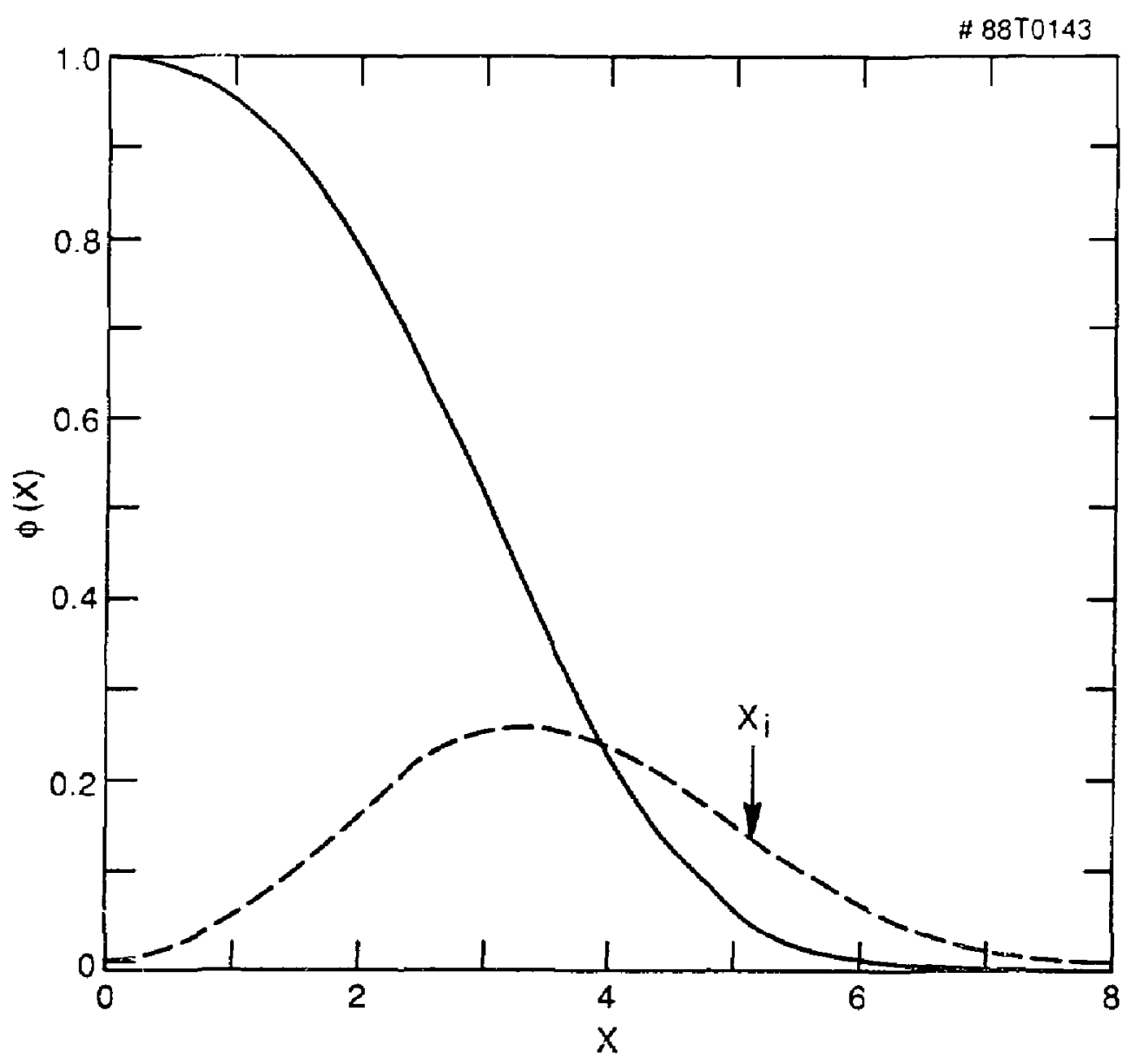

FIG. $8(b)$ 